\title{
Técnicas Alternativas de Ensino (Aprendizagem Ativa) para Disciplinas da Computação: Um Mapeamento Sistemático no Contexto Brasil
}

\author{
Ronney Moreira de Castro, Sean Wolfgand Matsui Siqueira \\ Programa de Pós-Graduação em Informática (PPGI) \\ Universidade Federal do Estado do Rio de Janeiro (UNIRIO) \\ Av. Pasteur, 456 - Urca - Rio de Janeiro - RJ - Brasil \\ ronney.castro@uniriotec.br, sean@uniriotec.br
}

\begin{abstract}
The present work presents a research on alternative techniques (of active learning) used for teaching subjects in the area of Computer Science. The investigation focus was restricted to the Brazilian context and it was performed through a systematic mapping of the literature. The works were classified based on categories of Active Learning techniques and of courses from the Computer Science area, according to the formation referentials. As a result, a concentration was observed in techniques related to technological interaction and on algorithms and programming techniques courses.
\end{abstract}

Resumo. O presente trabalho apresenta uma pesquisa sobre técnicas alternativas (de aprendizagem ativa) utilizadas no ensino de disciplinas da área da Computação. O foco da investigação foi restrito ao contexto brasileiro e foi realizado através de um mapeamento sistemático da literatura. Os trabalhos encontrados foram classificados com base em categorias de técnicas de Aprendizagem Ativa e de disciplinas da área de Computação, conforme os referenciais de formação. Como resultado, foi observada uma concentração em técnicas relacionadas à interação tecnológica e em disciplinas de algoritmos e técnicas de programação.

\section{Introdução}

O mundo moderno evolui cada vez mais e torna-se dependente de diversas tecnologias. Recursos que antes eram escassos ou mesmo inexistentes, como os Smartphones, fazem parte do dia-a-dia das pessoas [Posch; Fitzpatrick 2012]. Este cenário também está dentro das salas de aula. O perfil antigo, no qual os alunos estavam acostumados a ficar em seus lugares, anotar/escutar o que o professor passava nas aulas, mudou. A aula expositiva ainda é o modelo mais utilizado por grande parte dos docentes. No entanto, este tipo de aula pode não ser o método mais eficaz [Acharya et al. 2015].

Diante desse cenário um número crescente de estratégias vem ganhando destaque no que diz respeito a obter a atenção dos alunos, mudando a forma tradicional de ensino e aprendizagem. Alguns exemplos são: salas de aula invertida, casos interativos e aprendizagem baseada em problemas [Mitchell; Petter; Harris 2017]. A Aprendizagem Ativa (AA) é uma estratégia que vem provando ser válida no auxílio aos alunos em sua aprendizagem [Meyers; Jones 1993]. Suas técnicas complementam as aulas, motivam os discentes para um estudo mais detalhado, além de proporcionar uma maior retenção do conhecimento [Acharya et al. 2015]. 
VIII Congresso Brasileiro de Informática na Educação (CBIE 2019)

Anais do XXV Workshop de Informática na Escola (WIE 2019)

Este artigo traz como contribuição os resultados de um Mapeamento Sistemático realizado nos principais eventos relacionados à Informática na Educação e Ensino de Computação no Brasil nos últimos seis anos que mostram usos de técnicas alternativas de ensino para disciplinas relacionadas à Computação. A Seção 2 apresenta o que é AA do ponto de vista da literatura especializada; a Seção 3 apresenta uma breve descrição sobre os Referenciais de Formação da Sociedade Brasileira de Computação que serão adotados nesse artigo, a Seção 4 traz a metodologia utilizada nesse trabalho; a Seção 5 exibe alguns dos resultados do Mapeamento Sistemático. Por fim, a seção 6 traz as considerações finais.

\section{Aprendizagem Ativa}

Existem diversos conceitos na literatura especializada para Aprendizagem Ativa, entre os quais pode-se destacar: "Qualquer movimento que envolve os alunos em fazer as coisas e pensar sobre o que estão fazendo" [Bonwell; Eison 1991]; "Qualquer método que envolva os alunos no processo de aprendizagem" [Prince 2004]; "Atividades instrucionais envolvendo os alunos a fazerem coisas e pensar sobre o que estão fazendo" [Massey; Brown; Johnston, 2005]. Mitchell; Petter e Harris (2017) atualizaram estas definições ao afirmar que AA "são exercícios introduzidos na sala de aula para incentivar o pensamento e a participação dos alunos em um esforço para envolvê-los no processo de aprendizagem". Em geral, os elementos principais da AA são a atividade dos discentes e seu engajamento no processo de aprendizagem [Prince 2004]. A AA permite aos alunos uma maior prática, ou seja, fazer mais do que apenas ouvir, participando de atividades, discussões, reflexões, tanto em sala de aula quanto fora dela [Massey; Brown; Johnston 2005].

\section{Referenciais de Formação da Sociedade Brasileira de Computação}

A Sociedade Brasileira de Computação (SBC) vem trabalhando, desde 2010, na reformulação do Currículo de Referência (CR) dos cursos de Computação. Em dezembro de 2017 foi disponibilizado para a comunidade o livro "Referenciais de Formação para os Cursos de Graduação em Computação" (RFs), que contém os referenciais de formação para os cursos da área de Computação [Zorzo et al. 2017].

Os RFs não são currículos prontos, mas sim um material de consulta para sua elaboração. Entretanto, é possível verificar no livro que existem conteúdos variados para os cursos da área. Para este trabalho, foi feita uma compilação desses diversos conteúdos, que foi utilizada no Mapeamento Sistemático para classificação das disciplinas envolvidas na área.

\section{Metodologia}

O Mapeamento Sistemático da Literatura (MSL) é um tipo específico de Revisão Sistemática com uma visão mais ampla dos estudos primários, muito utilizado quando o cenário é abrangente e cujo objetivo é reunir o máximo de informações sobre determinada área de estudo [Kitchenham; Charters 2007]. Nesse trabalho, foi conduzido um MSL com objetivo de verificar técnicas alternativas (Técnicas AA) para o ensino de disciplinas de cursos de Computação no contexto brasileiro, mais especificamente responder a seguinte questão de pesquisa $(\mathrm{QP})$ : “Quais são as técnicas alternativas de 
VIII Congresso Brasileiro de Informática na Educação (CBIE 2019)

Anais do XXV Workshop de Informática na Escola (WIE 2019)

ensino (Técnicas de Aprendizagem Ativa) utilizadas nas diversas disciplinas dos cursos da área de Computação no contexto brasileiro?"

Como a QP se restringe ao universo brasileiro, a pesquisa foi realizada através de busca nos anais dos principais eventos do país relacionados à Informática na Educação e Ensino de Computação: CBIE; SBIE; WEI; WIE, além da revista RBIE, nos anos 2013 a 2018. Os critérios de inclusão e exclusão para o MSL estão representados na Tabela 1. A tabela 2 exibe a quantidade total de trabalhos de cada evento/revista separados por ano e os que foram incluídos no MSL após aplicação dos critérios de inclusão e exclusão.

Tabela 1: Critérios de inclusão e exclusão.

\begin{tabular}{|c|c|}
\hline ão & Crit \\
\hline $\begin{array}{l}\text { - Trabalho que mencione uso de tec. alternativas } \\
\text { para ensino de disciplinas de computação; } \\
\text { - Trabalho que mencione uso de metodologias } \\
\text { alternativas para ensino de disciplinas de } \\
\text { computação; } \\
\text { - Trabalho que mencione uso de ferramentas para } \\
\text { - } \quad \text { Trabalhos relacionados à educação básica; } \\
\text { - Trabalhos relacionados ao ensino técnico; } \\
\text { - Trabalhos relacionados ao ensino superior. }\end{array}$ & $\begin{array}{l}\text { - Trabalhos que estejam relacionados a disciplinas } \\
\text { que não sejam da área de computação; } \\
\text { Trabalhos que não estejam ligados a técnicas e/ou } \\
\text { metodologias para ensino de disciplinas de } \\
\text { computação; } \\
\text { - Trabalhos que estejam focados em algum gênero } \\
\text { específico; } \\
\text { - Trabalhos que estejam relacionados a culturas } \\
\text { específicas e/ou etnias; } \\
\text { Mapeamentos e Revisões Sistemáticas. }\end{array}$ \\
\hline
\end{tabular}

Tabela 2: Total de trabalhos nos eventos e na revista RBIE (coluna Total) e incluídos no MSL (Coluna MSL).

\begin{tabular}{|c|c|c|c|c|c|c|c|c|c|c|c|c|c|c|}
\hline \multirow{2}{*}{$\begin{array}{c}\text { Evento } \\
\text { Ano }\end{array}$} & \multicolumn{2}{|c|}{2013} & \multicolumn{2}{|c|}{2014} & \multicolumn{2}{|c|}{2015} & \multicolumn{2}{|c|}{2016} & \multicolumn{2}{|c|}{2017} & \multicolumn{2}{|c|}{2018} & \multicolumn{2}{|c|}{ Total Geral } \\
\hline & Total & MSL & Total & MSL & Total & MSL & Total & MSL & Total & MSL & Total & MSL & Total & MSL \\
\hline CBIE & 91 & 4 & 85 & 5 & 153 & 17 & 159 & 20 & 152 & 10 & 128 & 14 & 768 & 70 \\
\hline RBIE & 30 & 1 & 33 & 3 & 44 & 2 & 33 & 0 & 17 & 2 & 20 & 3 & 177 & 11 \\
\hline SBIE & 114 & 5 & 162 & 12 & 140 & 8 & 133 & 14 & 172 & 7 & 171 & 12 & 892 & 58 \\
\hline WEI & 54 & 18 & 44 & 14 & 45 & 14 & 47 & 19 & 25 & 10 & 52 & 24 & 267 & 99 \\
\hline WIE & 52 & 4 & 71 & 4 & 67 & 6 & 104 & 10 & 133 & 19 & 77 & 3 & 504 & 46 \\
\hline TOTAL & 341 & 32 & 395 & 38 & 449 & 47 & 476 & 63 & 499 & 48 & 448 & 56 & 2.608 & 284 \\
\hline
\end{tabular}

\section{Resultados do MSL}

A distribuição temporal e por tipo de ensino dos artigos incluídos na revisão é apresentada na Figura 1. Percebe-se que a comunidade científica brasileira vem mantendo o interesse por pesquisas relacionadas a técnicas alternativas de ensino de disciplinas para Computação, principalmente nos ensinos Básico e Superior.

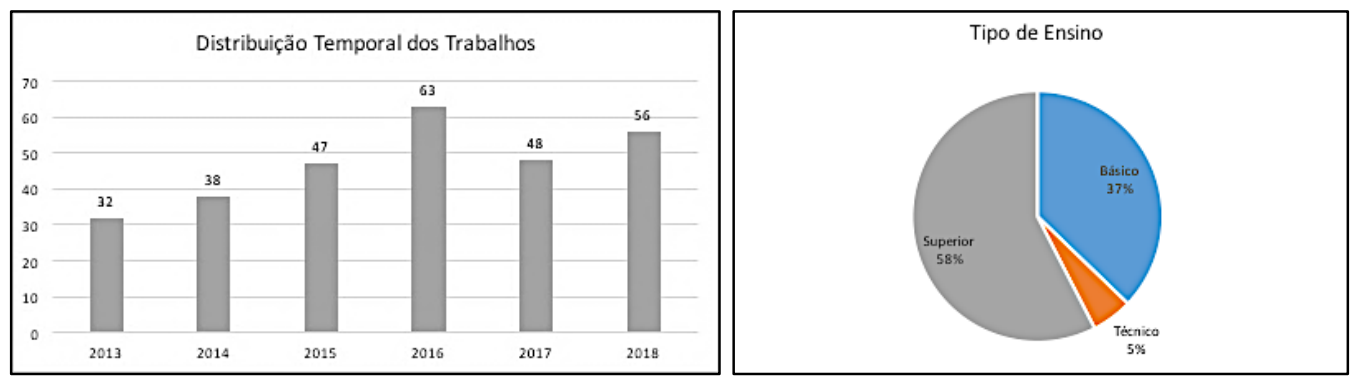

Figura 1. Gráfico de distribuição dos artigos 
VIII Congresso Brasileiro de Informática na Educação (CBIE 2019)

Anais do XXV Workshop de Informática na Escola (WIE 2019)

Foram encontradas 53 técnicas diferentes e, a maioria delas, está em conceitos iniciais da Computação, como "Algoritmos" e "Técnicas de Programação", conforme ilustra a figura 2 .

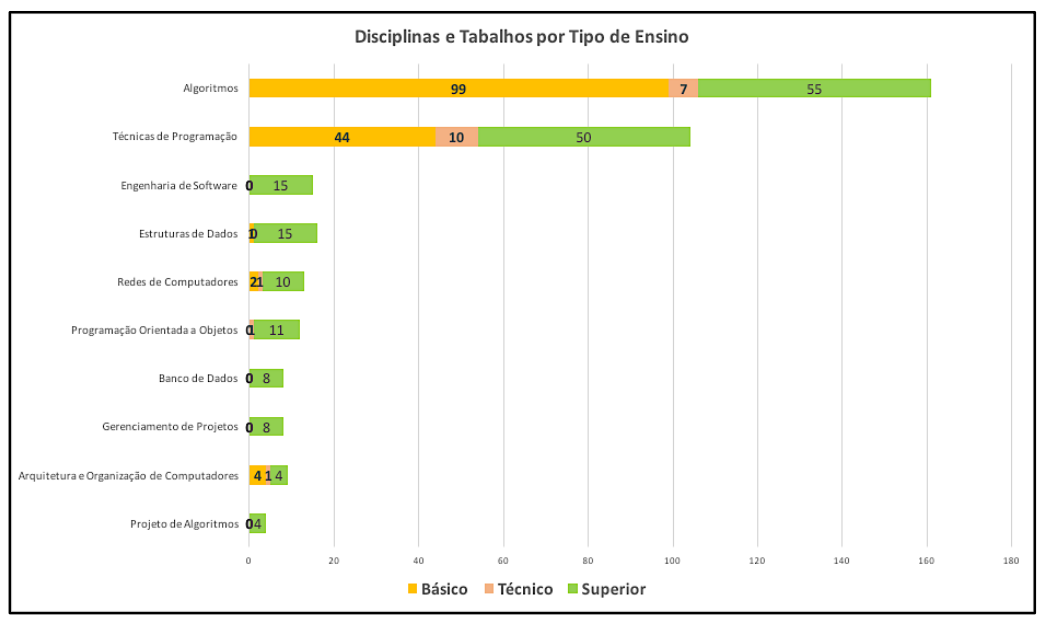

Figura 2. Gráfico de disciplinas e trabalhos por tipo de ensino

Hoje existe uma preocupação com a grande evasão que ocorre nos cursos superiores em Computação, muitas das vezes relacionada ao não entendimento de conceitos básicos [Soares et al. 2016]. A SBC, bem como outras entidades, tem trabalhado para inserir a Computação no ensino básico [DCEB 2019]. Pelos resultados apresentados no MSL verifica-se que existe uma quantidade de técnicas AA considerável que pode ser utilizada por docentes para ensinar conceitos de Computação para alunos do ensino básico, como "Algoritmos", por exemplo, embora a Computação no ensino básico não esteja restrita ao ensino de algoritmos e programação. A própria SBC vem trabalhando nessa questão com a criação da Diretoria Extraordinária de Ensino de Computação na Educação Básica da SBC, discussões em audiências públicas sobre a BNCC e palestras promovidas sobre o tema.

De forma a apoiar o professor a encontrar estas técnicas descritas nos artigos, conforme os conteúdos categorizados para a área de Computação, foi desenvolvido o portal ALCASYSTEM' [Castro; Siqueira, 2019].

\section{Conclusões}

As aulas tradicionais (expositivas) tendem a não prender a atenção dos alunos e, o uso de técnicas alternativas que permitam uma melhor motivação, passa a ser um diferencial. Entretanto, os desafios de adotar uma nova técnica podem levar o docente a desistir [Hirdes et al. 2006]. O uso das técnicas AA envolve uma mudança cultural por parte dos docentes. Um direcionamento para o planejamento de atividades didáticopedagógicas para as aulas de Computação pode incluir técnicas de AA para apoiar o ensino e aprendizagem.

Embora possam ser observadas limitações na adoção de AA como, por exemplo, a necessidade de recursos específicos ou preparação dos professores, exercícios baseados em AA oferecem mecanismos adicionais para que docentes e alunos possam construir novos cenários de aprendizagem. Nesse trabalho também foi possível verificar

\footnotetext{
${ }^{1}$ Disponível em: https://goo.gl/7HNUxj
} 
VIII Congresso Brasileiro de Informática na Educação (CBIE 2019)

Anais do XXV Workshop de Informática na Escola (WIE 2019)

que tais técnicas têm sido utilizadas no contexto brasileiro e, com isto, estratégias de disseminação destas práticas podem ser elaboradas. Espera-se também que com o uso dessa metodologia seja possível melhorar o ensino em Computação e, consequentemente minimizar a evasão dos cursos na área.

\section{Referências}

Acharya, S., Manohar, P., Wu, P. Y., Ansari, A. A., Schilling, W. W. (2015). Integrated Active Learning Tools for Enhanced Pedagogy in a Software Engineering Course Paper presented at 2015 ASEE Annual Conference \& Exposition, Seattle, Washington.

Bonwell, C. C., Eison, J. A. (1991). Active learning: Creating excitement in the classroom. Washington, DC: Association for the Study of Higher Education.

Castro, R. M., Siqueira, S. W. M. (2019). ALCASYSTEM - Active Learning In Computer Science - Um Portal com Técnicas de Aprendizagem Ativa para Disciplinas da Área da Computação. Apps.edu. Anais dos Workshops do Congresso Brasileiro de Informática na Educação, 2019.

DCEB - Diretrizes para ensino de Computação na Educação Básica. (2019). Disponível em:<http://www.sbc.org.br/educacao/diretrizes-para-ensino-de-computacao-naeducacao-basica>. Acesso em: 29 jun. 2019.

Hirdes, J. C. R., Souza, J. A., Dandolini, G. A., Mello, J., Rodrigues, J. M. (2006). Monitoria em vídeo: o uso das novas tecnologias de comunicação no processo de ensino-aprendizagem. EGEM - Encontro Gaúcho de Educação Matemática.

Kitchenham, B. e Charters, S. (2007). Guidelines for performing systematic literature reviews in software engineering. Technical report, Keele University and Durham University Joint Report.

Massey, A. P., Brown, S. A., Johnston, J. D. (2005). It's All Fun and Games. Until Students Learn. Journal of Information Systems Education, 16 (1).

Meyers, C., Jones, T. (1993). Promoting active learning: Strategies for the college classroom. San Francisco, CA: Jossey- Bass Publishers.

Mitchell, A., Petter, S., Harris, A. (2017). Learning By Doing: Twenty Successful Active Learning Exercises for Information Systems Courses. Journal of Information Technology Education: Innovations in Practice, 16(3), 21-46.

Prince, M. (2004). Does active learning work? A review of the research. Journal of Engineering Education, 93(3), 223-231. Pundak.

Posch, I., Fitzpatrick, G. (2012). First steps in the FabLab. Proceedings of the 24th Australian Computer-Human Interaction Conference on - OzCHI '12, 497-500.

Soares, J. P. R. S., Cercil, R. G., Monte-Alto, H. H. L. C. (2016). Clube de programação e oficinas como Scratch: um relato de experiência. Anais dos Workshops do V Congresso Brasileiro de Informática na Educação (CBIE 2016), p. 958-962.

Zorzo, A. F., Nunes, D., Matos, E., Steinmacher, I., Leite, J., Araujo, R. M., Correia, R., Martins, S. (2017). Referenciais de Formação para os Cursos de Graduação em Computação. Sociedade Brasileira de Computação (SBC). 153p, 2017. 